\title{
La Incapacidad laboral como indicador de gestión sanitaria
}

\section{Labour disability as healthcare management indicator}

\author{
José Manuel Vicente Pardo' \\ 1. Unidad Médica Equipo Valoración Incapacidades Gipúzkoa. INSS. España \\ Recibido: 16-06-15 \\ Aceptado: 23-06-15

\section{Correspondencia} \\ José Manuel Vicente Pardo \\ Pescadores Gran Sol, s/n \\ San Sebastián 20011, Gipúzkoa. España. \\ Correo electrónico: jose-manuel.vicente@seg-social.es \\ josemanuvicente@gmail.com
}

Resumen

La incapacidad laboral es la situación de pérdida de la capacidad laboral consecuencia de las limitaciones funcionales derivadas de enfermedad o lesión sobrevenida, por lo que está relacionada de forma primordial y directa con la pérdida de la salud (limitación funcional con impedimento laboral), ya que sin esta pérdida no cabría la determinación de una situación de incapacidad. Por ello la incapacidad laboral tiene un elemento sanitario que conviene conocer y los datos derivados de la misma son útiles tanto por su valor como indicador de resultado de las acciones sanitarias, como por su referente como indicador de calidad de vida, pues su situación establece una merma de la calidad de vida básica y fundamentalmente la utilización de los datos de incapacidad como indicador para la gestión sanitaria de gran trascendencia y que obliga al tratamiento de la misma más allá de la simple cuantificación economicista que la priva de la trascendencia sanitaria que dimana de sus datos de salud la incapacidad laboral relacionados, incluyendo el elemental de la enfermedad o la lesión que se padece. El uso de los datos de incapacidad con fines de gestión exige el soporte informático adecuado que la facilite y dada su enorme escala y diversidad, ello sólo es posible con el uso de herramientas de tecnología informática como los «big data» que permitan la recogida, el archivo, comunicación, transferencia y análisis de los datos de incapacidad de enorme volumen y complejidad y su interrelación con otros para confeccionar el mapa de la incapacidad y convertirse en herramienta de planificación y estrategia de gestión sanitaria y de protección social. No obstante lo cual, y hasta que ello no esté disponible, es imprescindible y beneficioso, el uso del dato de incapacidad tipo y grado, asociado a diagnóstico y actividad laboral como un indicador de gestión.

Conclusiones: La incapacidad es un indicador de resultado sanitario, un indicador de calidad de vida y un indicador de gestión sanitaria. Hay que avanzar en la recogida, archivo y utilización de los datos de incapacidad más allá de las referencias al coste o al número o distribución geográfica o por regímenes de aseguramiento, para una mejora de la información, el tratamiento informatizado de las bases de datos y su explotación epidemiológica, con la finalidad de facilitar la comunicación de información sanitaria, estableciendo acuerdos institucionales que permitan compartir archivos/información en garantía de la mejor protección ciudadana en materia de salud. Es necesario dar respuesta a las demandas de datos sobre incapacidad que desde las entidades, las direcciones y las jefaturas de gestión sanitaria, así como de las unidades de gestión clínica y de los servicios clínicos, se formulan, máxime por la enorme utilidad que del conocimiento de los datos sobre incapacidad de un determinado proceso y su área de presentación pueden reportar como indicador de resultado sanitario y como instrumento de ayuda a la toma de decisiones en la gestión, tanto clínica (microgestión) como sanitaria (macro y mesogestión).

Med Segur Trab (Internet) 2015; 61 (239) 207-219 
Palabras clave: incapacidad laboral, indicador de gestión sanitaria, datos incapacidad laboral, big data en gestión sanitaria.

Abstract

Labor disability is defined as the loss of competence due to functional limitations caused by disease or injury. That is why it is primarily and directly related with a loss of health (functional limitation with loss of working capacity). Without this loss, a disability determination cannot be applied. Therefore it is convenient to know the health component of labor disability. This data provided is useful not only for its value to determine the result of the health actions, but also as a quality-of-life indicator. Since this situation means a decrease in basic standard of living, the use of disability data as a health management indicator is of great significance. Consequently, the disability treatment is considered beyond any simple economic criteria that hinder its medical importance resulting from the labor disability related data -including the nature of the disease or the injury suffered. The use of the disability data for management requires the appropriate software that can ease the task. Given its huge scale and diversity, only software tools such as the big data can ensure the compilation, archive, communication, transfer and the disability data analysis of those large and complex files. With such software, data can be interlinked, allowing to map the disability and becoming a planning and strategic health management tool and social prevention. It is essential and beneficial the use of data type and degree disability associated with labor activity diagnosis and management as an indicator.

Conclusions: Disability is an indicator of health outcome, an indicator of quality of life and a healthcare management indicator. We must move forward in the collection, storage and use of disability data beyond references to the costs, number or geographic distribution or assurance schemes, in order not only to improve the information processing of computer databases and epidemiological exploitation but to facilitate the communication of health information, establishing institutional arrangements for sharing files / information and thus securing the best public health protection. It is necessary to meet the demands of data on disability that from entities, addresses and health management headquarters and units of clinical management and clinical services appeared, especially by the enormous usefulness of knowledge data on disability in a given process and its display area that can report as an indicator of health outcome and as an aid to take decisions in the management of both clinical (micro) and health (macro and middle management).

Med Segur Trab (Internet) 2015; 61 (239) 207-219

Keywords: Disability, healthcare management indicator, data disability for work, big data in health management. 


\section{INTRODUCCIÓN}

La incapacidad laboral es la situación de pérdida de la capacidad laboral consecuencia de las limitaciones funcionales derivadas de enfermedad o lesión sobrevenida.

Concepto de incapacidad temporal: Tendrán la consideración de situaciones determinantes de incapacidad temporal las debidas a enfermedad común o profesional y a accidente, sea o no de trabajo, mientras el trabajador reciba asistencia sanitaria de la Seguridad Social y esté impedido para el trabajo, con una duración máxima de trescientos sesenta y cinco días, prorrogables por otros ciento ochenta días cuando se presuma que durante ellos puede el trabajador ser dado de alta médica por curación. Artículo 128 TRLGSS (Texto Refundido Ley General de Seguridad Social).

Concepto de incapacidad permanente: la situación del trabajador que, después de haber estado sometido al tratamiento prescrito, presenta reducciones anatómicas o funcionales graves, susceptibles de determinación objetiva y previsiblemente definitivas, que disminuyan o anulen su capacidad laboral. No obstará a tal calificación la posibilidad de recuperación de la capacidad laboral del inválido, si dicha posibilidad se estima médicamente como incierta o a largo plazo.

Así las cosas la incapacidad tanto temporal como permanente está relacionada de forma primordial con la pérdida de la salud (limitación funcional con impedimento laboral), ya que sin esta pérdida no cabría la determinación de una situación de incapacidad.

Por ello la incapacidad tiene un elemento sanitario derivado de la necesidad de seguir tratamiento de forma temporal para conseguir la recuperación o de su de ineficacia condicionando pérdidas funcionales o anatómicas que disminuyen o anulan la capacidad laboral de forma permanente, y derivado de que en su comprobación es necesario el reconocimiento médico, así como en su valoración funcional.

El carácter económico de la incapacidad, es el derivado de la cobertura económica y la protección social de la situación de necesidad derivada de la pérdida de la capacidad de ganancia por pérdida de salud con carácter incapacitante laboral.

Es cierto que para tener derecho a la incapacidad se precisan otros condicionantes no sanitarios, referentes a carencia (cotización a la seguridad social), edad, alta en seguridad social, etc.

Pero si el componente sanitario es esencial en el reconocimiento de la incapacidad laboral, resulta básico el conocimiento y el análisis de cuantos factores condicionan la aparición, la evolución y el deterioro de la pérdida de salud permanente y con ello la pérdida o anulación de la capacidad laboral, bien fuera temporal (baja) como permanente.

Por otra parte estar incapacitado es estar limitado a pesar de seguir tratamiento, lo que supone la necesidad y el uso de recursos sanitarios.

Dicho esto resulta sorprendente que los datos que se disponen o que se presentan públicamente sobre la incapacidad temporal o permanente hacen mención al componente económico exclusivamente. Conocemos el gasto en pensiones de incapacidad permanente o el gasto en incapacidad temporal, pero no se conocen los datos referentes a sus causas las enfermedades o lesiones que las causan, lo que sería lo primero que debiéramos de conocer pues lo más importante es porque se incapacita o porque se llega a esa situación de incapacidad, y no sólo cuánto cuesta a la seguridad social su protección. Por último en esta semblanza general del valor de los datos referentes a incapacidad laboral, obvio es decir que la situación de incapacidad conlleva importante consumo de recursos sanitarios, económicos y prestacionales y así mismo tiene una repercusión personal, social, económica y laboral tremenda. 


\section{La incapacidad datos}

Los datos de incapacidad son algo más que su situación, es decir, si hay o no incapacidad temporal o permanente y si es esta última, grado de la misma. Pues al ser datos de salud, precisamos el diagnóstico y todas aquellas variables de salud, que modulan la misma, actividad laboral, edad, sexo, situación de empleo, limitaciones funcionales presentes, diagnósticos secundarios incapacitantes, tratamientos, etc.

La multiplicidad de datos adosados al dato de incapacidad, su presencia en diversos formatos y diferentes archivos y presentación, (informes médicos de evaluación, documentación clínica, dictámenes, resoluciones) así como el inmenso volumen de los mismos y complejidad, sin olvidar el respeto a las medidas de confidencialidad en materia extremadamente sensible como son los datos de salud, hacen necesario el uso de sistemas Big Data, para su gestión y análisis de esa enorme magnitud de datos que no pueden ser tratados de manera convencional, ya que superan los límites y capacidades de las herramientas de software habitualmente utilizadas para la captura, gestión y procesamiento de datos.

No obstante lo cual, y hasta que ello no esté disponible, es imprescindible y beneficioso, el uso del dato de incapacidad tipo y grado, asociado a diagnóstico y actividad laboral como un indicador de gestión.

Sin descuidar que la valoración del dato de incapacidad sirve para el análisis actuarial del riesgo, que aun siendo una protección social obligada por el sistema de seguridad social, conviene conocer cuando se valora la sostenibilidad del sistema o analizar el fenómeno y sus cambios históricos o evolutivos, su comportamiento asociado a otras circunstancias normativas, económicas, laborales o sociales, en permanente variabilidad.

\section{La Incapacidad como indicador}

La incapacidad como:

- Indicador de resultado.

- Indicador de calidad de vida.

- Indicador de gestión sanitaria.

\section{La incapacidad como Indicador de resultado}

Conocer los días consumidos por absentismo por Baja (IT) por diferentes procesos de enfermedad nos puede servir como medida de resultado de la atención sanitaria prestada a un determinado proceso o conjunto de procesos o grupo diagnóstico. Incorporando la incapacidad a los indicadores de salud (Performance Indicators).

Entendiendo que prestamos asistencia sanitaria con el objetivo maximalista de reparar la pérdida de salud y reintegrar al enfermo a su vida normalizada, lo más temprano posible, y que por vida normalizada como no debemos incluir la normalización de la vida laboral. Cuando valoramos resultados en salud, se busca mejorar la efectividad de la práctica clínica, la seguridad, la sensibilidad a las necesidades y la eficiencia de la atención sanitaria.

Conocer los días de baja o si finalmente fueron incapacitados de forma permanente los pacientes atendidos en un centro o un área, o un SPS, nos puede reportar un indicador por proceso que sirva no sólo para conocer el resultado de la acción sanitaria, sino para comparar nuevas terapias o cambios en la gestión sanitaria y en qué medida suponen mejora en los datos de incapacidad o relacionarlos con otros datos de gestión sanitaria o comparativas por centros, o por áreas o por servicios o entre los diferentes SPS.

Así como valoramos en el cáncer la remisión completa y los tiempos consumidos hasta lograrla, y es factor de buen pronóstico el menor tiempo en conseguirla, como indicador de eficacia terapéutica, nos serviría como indicador de buen resultado los días 
consumidos por baja hasta el alta por curación o mejoría suficiente y cuanto antes lleguemos a la normalización o restitución de la capacidad laboral mayor será la eficacia de los tratamientos aplicados. De hecho, servicios como los de rehabilitación cardiaca establecen la bondad del tratamiento en función de la recuperación laboral. Lo mismo sucede cuando se realiza estudio de pacientes en rehabilitación tras procesos osteomioarticulares, donde se recoge si ha existido reintegro a la vida laboral y en cuanto tiempo, y así mismo y como meros ejemplos cuando valoramos procesos oncológicos y precisamos conocer si tras el tratamiento se ha producido o no una retorno al trabajo como algunos estudios sobre cáncer de mama o procesos hematológicos se vienen realizando.

La obtención del dato de incapacidad por proceso incapacidad y diagnóstico) con la finalidad de estudiar las respuestas terapéuticas o la evaluación pronóstica o el análisis epidemiológico basado en la evidencia científica, puede realizarse mediante encuesta o formulario de autocumplimentación, si bien lo correcto sería el acceso a los archivos o la trasmisión de paquete de datos desde el sistema de seguridad social, lo que es complejo y engorroso, cuando no imposible.

\section{La incapacidad como Indicador de calidad de vida}

Los indicadores de calidad de vida hacen referencia al impacto de la enfermedad y su tratamiento sobre todos los aspectos de la vida del paciente, incorporando la perspectiva del propio paciente Entre los indicadores de calidad de vida y como un parámetro indispensable en ellos estaría la incapacidad resultante de una enfermedad, por las limitaciones consecuentemente derivadas de esta, y cuanto comporta para la percepción que de ello tiene el trabajador que se encuentra en situación de incapacidad laboral.

Entendiendo además la capacidad laboral y el trabajo como un derecho a proteger y un derecho universal.

Cuando valoramos la calidad de vida relacionada con la salud (CVRS) los instrumentos genéricos que habitualmente usamos para la evaluación del paciente son fundamentalmente:

- Cuestionario de Salud SF-36. ${ }^{\text {a }}$ valora función física, función social, rol físico, rol emocional, salud mental, vitalidad, dolor corporal, intensidad del dolor y su efecto en el trabajo habitual, salud general.

- EuroQol-5D: valora morbilidad, autocuidado, actividades habituales, dolor o malestar y ansiedad o depresión

- Viñetas COOP-WONCA: valora forma física, sentimientos, actividades sociales, actividades cotidianas, cambio en el estado de salud, estado de salud y dolor

- Perfil de Salud de Nottingham: valora energía, dolor, movilidad física, reacciones emocionales, sueño y aislamiento social

- Perfil de las consecuencias de la enfermedad SIP: valora Dimensión física (3 categorías) Dimensión psicosocial (4 categorías) y 5 categorías independientes.

La valoración de la incapacidad laboral como indicador de calidad de vida relacionada con la salud, debiera valorar la incapacidad y su grado (incapacidad total para su trabajo habitual, absoluta para todo tipo de trabajo, y gran invalidez), así como dimensiones afectas (al estilo de las referidas en la Ficha de Evaluación de Requerimientos del Foro de la Academia de Ciencias Médicas de Bilbao o las recogidas en la valoración según sistema MERCAL), valorando atención, precepción, comprensión oral y escrita, razonamiento, toma de decisiones, control emocional, relaciones sociales, insigth, capacidad manipulativa, auditiva, visual, dinámica. 


\section{La incapacidad como indicador de gestión}

Los datos de incapacidad tanto temporal como permanente nos pueden ser útiles en la gestión sanitaria, tanto en:

- Macrogestión sanitaria (política sanitaria).

- Mesogestión sanitaria (gestión de centros sanitarios).

- Microgestión sanitaria (gestión clínica).

En la macrogestión o la política sanitaria los datos de incapacidad temporal o permanente asociados específicamente a proceso o diagnóstico que motiva la misma, permitirían conocer cuáles son las causas de las situaciones incapacitantes, y establecer las medidas de prevención, y recursos sanitarios destinados a la recuperación temprana o el alivio de las dolencias incapacitantes. Si al diagnóstico añadimos trabajo entendiendo por trabajo ocupación, tareas, actividad de la empresa, riesgos o condiciones de trabajo podríamos establecer las adecuadas políticas de gestión en salud laboral.

El protocolo de Marco Estratégico para la mejora de la Atención Primaria en España 2007-2012, ya señalaba como elementos básicos el disponer de Indicadores de incapacidad temporal, conociendo el porcentaje de pacientes activos en incapacidad temporal por año, la duración media de las incapacidades temporales por áreas y procesos. Así como Identificar la interacción básica de las condiciones del trabajo y la salud para la adecuada prevención y manejo de las patologías prevalentes en salud laboral

En la mesogestión o gestión de centros o servicios los datos de incapacidad junto a otros nos pueden servir como referencia en la toma de decisiones o en la implementación de recursos y nuevas terapias o tecnologías sanitarias, así como el seguimiento y la eficacia de la gestión clínica mediante el manejo de resultados y atribuir recursos sanitarios y planificación estratégica. Incorporando a las decisiones o actuaciones clínicas análisis de resultados que serán de calidad si generan eficiencia económica y no mero control de gasto o tan sólo identificación del mismo.

En la microgestión o gestión clínica, los datos de incapacidad temporal, absentismo o tiempo consumido hasta la reincorporación laboral nos determinan el resultado terapéutico a igualdad de procesos y de circunstancias de paciente, y pueden ser extraordinariamente útiles el gestión de casos.

\section{Los datos económicos o numéricos de la incapacidad.}

A continuación expongo datos de elaboración propia en base a los que se disponen de libre acceso en la web de la seguridad social.

Teniendo en cuenta el número de bajas abiertas a marzo 2015 por cada mil trabajadores protegidos, por regímenes de seguridad social y por comunidades autónomas, podemos observar la diferente prevalencia ( $n .^{\circ}$ de procesos abiertos por mil trabajadores protegidos) tanto por comunidades autónomas como por regímenes de seguridad social. Es un indicador de duración y de impacto o presencia activa de la situación de absentismo. Que constata la diferente distribución de las bajas por comunidades autónomas.

Las comunidades autónomas de Galicia (siendo la que peores datos tiene), Cantabria, Murcia y Asturias son las que más bajas mantienen en trabajadores autónomos. Las cuatro que menos Ceuta, Madrid, Melilla, y Baleares (siendo la que mejores datos tiene).

Las comunidades autónomas de Galicia (siendo la que peores datos tiene), País Vasco, Canarias y Cantabria, son las que más bajas mantienen en trabajadores del resto de regímenes. Las cuatro que menos Andalucía, baleares, Madrid, la Rioja (siendo la que mejores datos tiene). 
Tabla 1. Datos a marzo 2015. Prevalencia IT $\mathrm{n}^{\circ}$ de procesos abiertos por mil trabajadores protegidos

\begin{tabular}{|c|c|c|c|}
\hline Comunidad Autónoma & $\begin{array}{c}\text { Prevalencia } \\
\text { Regímenes } \\
\text { sin autónomos }\end{array}$ & Comunidad Autónoma & Prevalencia Autónomos \\
\hline Galicia & 31,99 & Galicia & 40,51 \\
\hline País Vasco & 31,77 & Cantabria & 38,82 \\
\hline Canarias & 31,37 & Murcia & 38,51 \\
\hline Cantabria & 31,15 & Asturias & 37,55 \\
\hline Navarra & 30,88 & Castilla-La Mancha & 33 \\
\hline Asturias & 29,88 & Extremadura & 31,13 \\
\hline Murcia & 27,93 & Aragón & 29,34 \\
\hline Ceuta & 27,72 & Navarra & 29,03 \\
\hline Aragón & 27,21 & Com. Valenciana & 28,28 \\
\hline Cataluña & 26,96 & Castilla y León & 28,01 \\
\hline Castilla-La Mancha & 26,95 & Andalucía & 27,97 \\
\hline Melilla & 25,51 & Cataluña & 26,49 \\
\hline Com. Valenciana & 25,15 & La Rioja & 26,37 \\
\hline Castilla y León & 25,11 & País Vasco & 25,72 \\
\hline Extremadura & 24,62 & Canarias & 24,7 \\
\hline Andalucía & 24,14 & Ceuta & 21,59 \\
\hline Illes Balears & 24,12 & Madrid & 19,99 \\
\hline Madrid & 23,92 & Melilla & 19,02 \\
\hline La Rioja & 23,74 & Illes Balears & 18,94 \\
\hline Total Nacional & 26,47 & Total Nacional & 28,24 \\
\hline
\end{tabular}

La crisis ha afectado disminuyendo las bajas iniciadas pero no así su duración o su mantenimiento que ha aumentado. El coste ha disminuido pero, también ha disminuido el número de trabajadores. Parece en cualquier caso asistimos a un repunte de las bajas coincidiendo con la recuperación económica. El miedo a perder el empleo redujo el absentismo laboral a la mitad desde 2008, pasando del 3,10 en 2008 al 1,57 en 2013.

Si la distribución de las bajas por comunidad autónoma es tan dispar lo mismo sucede con la incapacidad permanente.

Si vamos a números absolutos de pensiones de incapacidad por comunidad autónoma los datos de la Tabla 2 a mayo 2015, referencian las comunidades con mayor número de pensiones y en la misma tabla las comunidades con mayor número de trabajadores protegidos. Puestas en relación vemos como aparentemente salvo algunas diferencias coinciden; como pareciera de esperar hay mayor número de pensiones en áreas con mayor número de trabajadores protegidos, pero es necesario conocer cuáles son las diferencias entre comunidades cuando lo trasladamos a su incidencia según trabajadores, es decir conocer en qué áreas geográficas hay mayor porcentaje de trabajadores incapacitados permanentes en relación con población trabajadora y es entonces cuando el panorama arroja algunas diferencias significativas, como se ve en la tabla 3. 
Tabla 2. Pensiones de IP, trabajadores protegidos por comunidades autónomas

\begin{tabular}{lrlc}
\hline & Pensiones de IP & & \multicolumn{2}{c}{ Trabajadores protegidos } \\
\hline ANDALUCÍA & 202.798 & CATALUNA & 3092393 \\
CATALUÑA & 164.883 & ANDALUCIA & 2904545 \\
C. VALENCIANA & 94.315 & MADRID & 2860758 \\
MADRID & 75.412 & C. VALENCIANA & 1687705 \\
GALICIA & 68.415 & GALICIA & 976608 \\
CASTILLA LEÓN & 44.540 & PAIS VASCO & 919714 \\
PAÍS VASCO & 42.456 & CASTILLA LEÓN & 873211 \\
C. LA MANCHA & 41.216 & CANARIAS & 727276 \\
CANARIAS & 34.089 & C. LA MANCHA & 640677 \\
MURCIA & 32.142 & MURCIA & 529760 \\
ASTURIAS & 31.691 & ARAGON & 524732 \\
EXTREMADURA & 24.475 & ILLES BALEARS & 415134 \\
ARAGÓN & 23.871 & EXTREMADURA & 378726 \\
ILLES BALEARS & 17.727 & ASTURIAS & 359006 \\
CANTABRIA & 13.502 & NAVARRA & 258658 \\
NAVARRA & 11.050 & CANTABRIA & 210878 \\
RIOJA (LA) & 5.042 & LA RIOJA & 123316 \\
CEUTA & 887 & MELILLA & 21579 \\
MELILLA & 1.152 & CEUTA & 21678 \\
\hline
\end{tabular}

En la Tabla 3, establecemos el porcentaje de incapacidades permanente por cada 100 trabajadores protegidos (afiliados) su coste y la tasa de paro por comunidad autónoma, en base a datos de la web de la seguridad social y del INE y elaboración propia a mayo 2015.

Tabla 3. Porcentaje de Incapacidad Permanente por trabajadores protegidos, tasa de paro y coste de IP

\begin{tabular}{lccc}
\hline \multicolumn{1}{c}{ CCAA } & $\begin{array}{c}\text { \% IP por trabajador } \\
\text { protegido }\end{array}$ & Tasa de paro & Coste de pensión \\
\hline ASTURIAS & $8.82 \%$ & 20,2 & $1.047,14$ \\
GALICIA & $7.00 \%$ & 18.8 & 747,03 \\
ANDALUCíA & $6.98 \%$ & 22,8 & 799,55 \\
EXTREMADURA & $6.46 \%$ & 24,8 & 741,17 \\
C. LA MANCHA & $6.43 \%$ & 22,4 & 820,56 \\
CANTABRIA & $6.40 \%$ & 19,7 & 925,42 \\
MURCIA & $6.06 \%$ & 18,7 & 780,02 \\
C. VALENCIANA & $5,58 \%$ & 20,1 & 815,62 \\
CATALUÑA & $5.33 \%$ & 14,1 & 917,31 \\
MELILLA & $5.33 \%$ & 34,0 & 874,66 \\
CASTILLA LEÓN & $5.10 \%$ & 17,5 & 867,86 \\
CANARIAS & $4.68 \%$ & 22,6 & 822,72 \\
PAÍS VASCO & $4.61 \%$ & 15,5 & $1.096,92$ \\
ARAGÓN & $4.54 \%$ & 14,8 & 926,28 \\
ILLES BALEARS & $4,27 \%$ & 10,0 & 815,37 \\
NAVARRA & $4.27 \%$ & 14,6 & $1.011,72$ \\
RIOJA (LA) & $4.08 \%$ & 14,5 & 856,52 \\
CEUTA & $4.09 \%$ & 36,1 & 874,66 \\
MADRID & $2,63 \%$ & 14,0 & $1.048,03$ \\
\hline
\end{tabular}


Como vemos en la tabla 3, las comunidades autónomas con mayor incidencia de incapacidad permanente por trabajador son Asturias, Galicia, Andalucía y Extremadura. Y las que menos Navarra, la Rioja, Ceuta y Madrid. Este dato es más relevante y de mayor significación por cuestionarnos su porqué, ya que el dato absoluto de pensiones de IP por comunidades autónomas, es un mero dato de cuantía o de valor económico, con esperados resultados más abundantes en poblaciones trabajadoras más voluminosas.

Entre diciembre de 2012 y junio de 2014, las pensiones de incapacidad permanente han pasado de 940.843 a 929.463 personas, 11.380 menos, según datos de la web seguridad social, lo que significa un descenso del 1,21\% en un año y medio, que sitúa los datos en cifras del 2009, cuando se registraron 930.831 pensionistas en situación de incapacidad permanente.

Según datos de mayo 2015 de los 929.663 pensionistas con un coste medio de $923,21 €, 32.473$ lo son en grado de Gran invalidez con un coste medio de $1.813,21 €$, 352.692 padecen una incapacidad permanente absoluta a $1.145 €$ de pensión media, 529.973 tienen una incapacidad permanente total para la profesión habitual a coste medio de $735 €$ y 235.111 una incapacidad permanente total cualificada (IPT más de 55 años y no trabajo) y 55 una incapacidad permanente parcial para la profesión habitual.

Andalucía es la comunidad autónoma con mayor número de personas en situación de incapacidad permanente, 202.798, seguida por Cataluña con 164.883 pensionistas y Comunidad Valenciana donde se encuentran 94.315 pensiones de incapacidad permanente. Madrid con 75.412 y Galicia con 68.415 sobrepasan los 50.000 pensionistas.

Más de la mitad de las pensiones de incapacidad permanente se dan en régimen general, 683.092 del total de 929.663, seguidas por los trabajadores autónomos, 124.883, y los accidentes de trabajado, 83.608 y 12.415 de enfermedad profesional. Por otro lado, el importe medio de la pensión se ha incrementado sensiblemente pasando de los $891,24 €$ de 2012 a los $923,21 €$ de mayo 2015, lo que supone un 3,58\% más.

En la Tabla 4 exponemos el coste de pensiones por comunidades autónomas, las pensiones más altas son País Vasco (tiene las pensiones más altas), Madrid, Asturias y Navarra y las más bajas Extremadura (tiene las pensiones más bajas), Galicia, Murcia y Andalucía.

Tabla 4. Coste medio de pensiones en cada comunidad autónoma

\begin{tabular}{lc}
\hline & Coste de pensión \\
\hline PAÍS VASCO & $1.096,92$ \\
MADRID & $1.048,03$ \\
ASTURIAS & $1.047,14$ \\
NAVARRA & $1.011,72$ \\
ARAGÓN & 926,28 \\
CANTABRIA & 925,42 \\
CATALUÑA & 917,31 \\
CEUTA & 874,66 \\
MELILLA & 874,66 \\
CASTILLA LEÓN & 867,86 \\
RIOJA (LA) & 856,52 \\
CANARIAS & 822,72 \\
C. LA MANCHA & 820,56 \\
C. VALENCIANA & 815,62 \\
ILLES BALEARS & 815,37 \\
ANDALUCÍA & 799,55 \\
MURCIA & 780,02 \\
GALICIA & 747,03 \\
EXTREMADURA & 741,17 \\
\hline
\end{tabular}


El coste, así como el número de pensiones, por grados de incapacidad en el global del sistema viene reflejado en la tabla 5 por grados de incapacidad.

Tabla 5. Grados de incapacidad permanente, número de pensiones y coste medio a mayo 2015

\begin{tabular}{c|c|c|c|c|c}
\hline $\begin{array}{c}\text { GI } \\
\mathbf{N} .^{\circ} \text { de pensiones } \\
\text { Coste medio }\end{array}$ & $\begin{array}{c}\text { IPA } \\
\mathbf{N}^{\circ} \text { de pensiones } \\
\text { Coste medio }\end{array}$ & $\begin{array}{c}\text { IPT } \\
\mathbf{N}^{\circ} \text { de pensiones } \\
\text { Coste medio }\end{array}$ & $\begin{array}{c}\text { IPT cualific. } \\
\text { N. }{ }^{\circ} \text { de pensiones } \\
\text { Coste medio }\end{array}$ & $\begin{array}{c}\text { IPP } \\
\text { N. }{ }^{\circ} \text { de pensiones } \\
\text { Coste medio }\end{array}$ & $\begin{array}{c}\text { Incap. Perm. } \\
\text { N. } .^{\circ} \text { de pensiones } \\
\text { Coste medio }\end{array}$ \\
\hline 32.473 & 352.692 & 294.862 & 235.111 & 55 & 929.663 \\
$1.813,21$ & $1.145,61$ & 606,95 & 896,54 & 158,65 & 923,21 \\
\hline
\end{tabular}

N. ${ }^{\circ}$ de pensiones y coste medio por grados de incapacidad (gran invalidez, absoluta, total, total cualificada, y parcial) a mayo 2015, datos extraídos de la sede electrónica de la seguridad social

Los datos por provincias a mayo 2015, reflejan que Barcelona ocupa el primer lugar en número de pensiones de incapacidad permanente, 127.445 , seguida por Madrid, con 75.412, Valencia, con 59.563, y Sevilla, con 54.859.

\section{La necesidad de conocer datos de incapacidad más allá de los datos econó- micos o numéricos}

En la actualidad los datos que conocemos son referentes a número de procesos de baja activos en un periodo, o su duración media y su distribución por comunidades autónomas y por provincias o por regímenes de afiliación a la seguridad social, pero desconocemos datos referentes a qué procesos causan las bajas con qué duración, a qué trabajadores afecta, y qué ocupación, tareas, o actividad o circunstancias de empleo se relaciona con cada proceso.

Los datos así considerados son meramente expositivos del número de bajas o cuánto duran globalmente consideradas; pero lo qué importa es conocer por qué se producen, qué tipo de enfermedades las provocan, cómo varían en función de los trabajos o de las edades o de la posibilidades terapéuticas.

\section{Necesidad de crear un mapa de la incapacidad en España}

Para analizar la población con situaciones de pérdida de salud, que determinan la necesidad de protección de social, por estar incapacitados, bien porque tienen reconocida una incapacidad contributiva o no contributiva, se necesita un conocimiento de los casos y sus causas.

Se precisa conocer el "riesgo asegurador", su coste de previsión y los recursos de «recuperación y reparación» del daño sobrevenido, así como la prevención del mismo.

Se precisa un "nodo" de intercambio, archivo e información de datos sobre la incapacidad, para una mejor utilización de los recursos sanitarios y económicoprestacionales, así como para la dotación presupuestaria a largo plazo, la contribución a la implementación de medidas de sostenibilidad del sistema y fijar políticas sanitarias, sociales y de incentivación de otras de necesaria realización personal, caso de insuficiencia de las públicas.

Lo cierto es que hasta ahora la seguridad social no dispone de un archivo del que poder extraer datos para conocer por qué tipo de procesos (enfermedad o lesiones) se otorgan o causan las incapacidades, tampoco es posible un análisis transversal comparando procesos y limitaciones con sectores de actividad y estos por grupos etarios, o sexo o incapacidades previas o tipo de trabajo o exposición a determinados riesgos, etc. Dicha situación de déficit de datos es concurrente tanto en la incapacidad permanente como en la temporal, y si bien es posible alguna explotación de datos esta sigue siendo muy deficiente. En el momento actual podemos conocer datos sobre el número de pensionistas, 
su coste, incluso su distribución por grados de incapacidad, geográficamente, edad y por sexo.

Si conocemos el impacto incapacitante de la pérdida de salud en la población trabajadora se procederá a una mejor gestión y adecuación de recursos atencionales, promocionales, preventivos, reintegradores, y prestacionales en torno a la incapacidad temporal y permanente, pero hoy por hoy no disponemos de este mapa, sabemos coste pero no causas, y la incapacidad es una prestación económica pero derivada de una situación de pérdida de salud y por ello con un componente sanitario y variables que determinan su aparición y evolución. Conocer nuestra población «incapacitada (por enfermedad)» es fundamental para cualquier análisis sobre impacto y necesidades de protección social, para contrastarla con la población activa, la población en formación o inactiva previa a la edad laboral, y la población inactiva jubilada.

Reducir las causas de la incapacidad laboral resulta una entelequia cuando se desconoce que es lo que causa su incapacidad. Y esto es importante también para actuar de forma preventiva y/o rehabilitadora sobre la población trabajadora o reparadora prestacional.

Es preciso compartir archivos entre instituciones y una reforma de los actuales que sin vulnerar la confidencialidad y seguridad de los mismos, permita por su volcado saber de la realidad de la incapacidad, más allá del número de pensiones o el coste de las mismas pues el dato económico no es suficiente.

La propuesta de creación de un mapa de la incapacidad implica por tanto determinar los datos básicos que deben constituir parte de la base de datos a explotar a futuro y establecer los mecanismos de registro, archivo, difusión y cómo compartir los mismos. Y esto sólo es posible si utilizamos sistemas informáticos basados en la acumulación a gran escala de datos y procedimientos para identificar patrones recurrentes dentro de esos datos, es decir mediante los denominaos Big Data o Datos Masivos que manipulan grandes conjuntos de datos y variables, su captura, almacenamiento, búsqueda, compartición, análisis y visualización.

El uso de los datos de incapacidad con fines de gestión exige el soporte informático adecuado que la facilite y dada su enorme escala y diversidad, ello sólo es posible con el uso de herramientas de tecnología informática como los «Big Data» que permitan la recogida, el archivo, comunicación, transferencia y análisis de los datos de incapacidad de enorme volumen y complejidad y su interrelación con otros para confeccionar el mapa de la incapacidad y convertirse en herramienta de planificación y estrategia de gestión sanitaria y de protección social. No obstante lo cual, y hasta que ello no esté disponible, es imprescindible y beneficioso, el uso del dato de incapacidad tipo y grado, asociado a diagnóstico y actividad laboral como un indicador de gestión, para establecer estrategias de mejora que permitan sistematizar y ordenar la atención sanitaria y la prestacional o de amparo social de forma adecuada y eficiente, sustentada en la mejor evidencia científica del análisis epidemiológico de los datos de incapacidad con el fin de ayudar a la toma de decisiones en distribución y mejora de recursos, con el fin de promover el conocimiento de la situación, establecer políticas para la prevención de la salud evitando en lo posible la aparición de las situaciones de incapacidad laboral, la atención sanitaria más correcta, y las medidas de rehabilitación socio laboral que faciliten y procuren la reincorporación laboral y cuando ello no sea posible la máxima protección a su situación de necesidad.

\section{CONCLUSIÓN}

La incapacidad es un indicador de resultado sanitario, un indicador de calidad de vida y un indicador de gestión sanitaria. Algo más que un indicador de gasto.

Es necesario dar respuesta a las demandas de datos sobre incapacidad que desde las entidades, las direcciones y las jefaturas de gestión sanitaria, así como de las unidades de 
gestión clínica y de los servicios clínicos, se formulan, máxime cuando esta demanda va en aumento entendiendo la enorme utilidad de conocer los datos sobre incapacidad de un determinado proceso y su área de presentación como indicador de resultado sanitario y de ayuda a la toma de decisiones en la gestión, tanto clínica (microgestión) como sanitaria (macro y mesogestión). Debemos utilizar los datos de incapacidad como herramienta de gestión. La incapacidad es un indicador de calidad de vida a incorporarlo a los que habitualmente utilizamos cuando valoramos la calidad de vida relacionada con la salud CVRS.

Hay que avanzar en la recogida, archivo y utilización de los datos de incapacidad más allá de las referencias al coste o al número o distribución geográfica o por regímenes de aseguramiento, para una mejora de la información, el tratamiento informatizado de las bases de datos y su explotación epidemiológica, con la finalidad de facilitar la comunicación de información sanitaria, estableciendo acuerdos institucionales que permitan compartir archivos/información en garantía de la mejor protección ciudadana en materia de salud. Es necesario dar respuesta a las demandas de datos sobre incapacidad que desde las entidades, las direcciones y las jefaturas de gestión sanitaria, así como de las unidades de gestión clínica y de los servicios clínicos, se formulan, máxime por la enorme utilidad que del conocimiento de los datos sobre incapacidad de un determinado proceso y su área de presentación pueden reportar como indicador de resultado sanitario y como instrumento de ayuda a la toma de decisiones en la gestión, tanto clínica (microgestión) como sanitaria (macro y mesogestión).

El Mapa de incapacidad con el objetivo de conocer más sobre las causas y el impacto de las situaciones de incapacidad es necesario para la gestión integral de la misma, para una mejor distribución de los recursos, para prevenir las situaciones de pérdida de salud y del quebranto por sus secuelas, facilitando y potenciando mecanismos de detección de la enfermedad y los aspectos preventivos de la salud laboral, homogeneizando criterios de valoración para una mayor garantía de protección social y mejorar la gestión sanitaria y la protección social basada en el conocimiento, con procedimientos tecnológicos tipo Big Data que aporten las máximas posibilidades de interrelación y análisis para la mayor trasparencia en la gestión, basada en la evidencia, optimizándola y aportando referencias para la mejor distribución de recursos y la ayuda a la toma de decisiones. Es urgente adaptar los sistemas de recogida de datos en incapacidad para conseguir una mejor explotación epidemiológica que facilite conocer la situación, su análisis y la planificación estratégica.

\section{BIBLIOGRAFÍA Y DOCUMENTACIÓN CONSULTADA}

Lizán T y Reig A. "La evaluación de la calidad de vida relacionada con la salud en la consulta: las viñetas COOP/WONCA". Atención Primaria 2002.

Esther Martín del Campo. "Big Data en salud, el camino hacia un cambio de paradigma en Medicina». Gaceta Médica noviembre. 2014.

Luis Joyanes Aguilar/Juan Miguel Poyatos Díaz. "Big Data y el sector de la salud: el futuro de la sanidad». www.poyatosdiaz.com.

Jordi Varela, Xavier Castells, Cristina Iniesta y Francesc Cots. «Instrumentos de la gestión clínica: desarrollo y perspectivas". Med. Clin. (Barc). 2008.

Peiró S, Meneu R. «Variaciones en la práctica médica: implicaciones para la práctica clínica y la política sanitaria». Gaceta Sanitaria. 1998.

Xavier Bonfill Asistencia Sanitaria Basada en la Evidencia: algunas propuestas para los partidarios de impulsar su formación. De la evidencia a la práctica clínica. Novartis.

José Manuel Vicente. Ponencia "La incapacidad laboral como Indicador en resultados en Salud relacionado con la vida laboral». III Foro Direcciones Médicas, mesa «Tecnologías de la información su influencia en la mejora de la Gestión Sanitaria». 9 Mayo 2015.

Jordi Varela «Datos al servicio de la salud». Blog "gestionclínicavarela». 
Juan Goiria Ormazábal «Ficha de valoración ocupacional para trabajadores en Incapacidad Temporal». Revista Medicina y Seguridad del Trabajo. 2014; Suplemento extraordinario n. ${ }^{\circ}$ 1. Instituto de Salud Carlos III.

Raúl Regal Ramos «El Médico Inspector de la Seguridad Social». Revista Medicina y Seguridad del Trabajo. 2014; Suplemento extraordinario n. ${ }^{\circ}$ 1. Instituto de Salud Carlos III.

III Informe Adecco sobre absentismo 2014

Web seguridad social sede electrónica. www.se-social.es.

Jornada Adegi 2011 «Los cambios en la incapacidad temporal». Datos de IT. www.adegi.es.

Instituto de Salud Carlos III Jornada "Oportunidades de Mejora en valoración médica de la incapacidad laboral». Modernización e integración de los sistemas de información, explotación epidemiológica. Junio 2014. www.eng.isciii.es/ISCIII. 